\title{
Raymond J. DeMallie (1946-2021) et Douglas R. Parks (1942-2021)
}

Un couple de savants exemplaires au cœur des Grandes Plaines

Emmanuel Désveaux et Gilles Havard

\section{(2) OpenEdition}

\section{Journals}

Édition électronique

URL : https://journals.openedition.org/jsa/19679

DOI : 10.4000/jsa. 19679

ISSN : $1957-7842$

\section{Éditeur}

Société des américanistes

Édition imprimée

Date de publication : 30 septembre 2021

Pagination : 219-228

ISSN : 0037-9174

Référence électronique

Emmanuel Désveaux et Gilles Havard, «Raymond J. DeMallie (1946-2021) et Douglas R. Parks (1942-2021) », Journal de la Société des américanistes [En ligne], 107-1 | 2021, mis en ligne le 30 septembre 2021, consulté le 03 septembre 2022. URL : http://journals.openedition.org/jsa/19679 ; DOl : https://doi.org/10.4000/jsa. 19679

Ce document a été généré automatiquement le 3 septembre 2022

Tous droits réservés 


\section{Raymond J. DeMallie (1946-2021) et Douglas R. Parks (1942-2021)}

Un couple de savants exemplaires au cœur des Grandes Plaines

\section{Emmanuel Désveaux et Gilles Havard}

Fig. 1 - Raymond J. DeMallie, Savoie, 2011

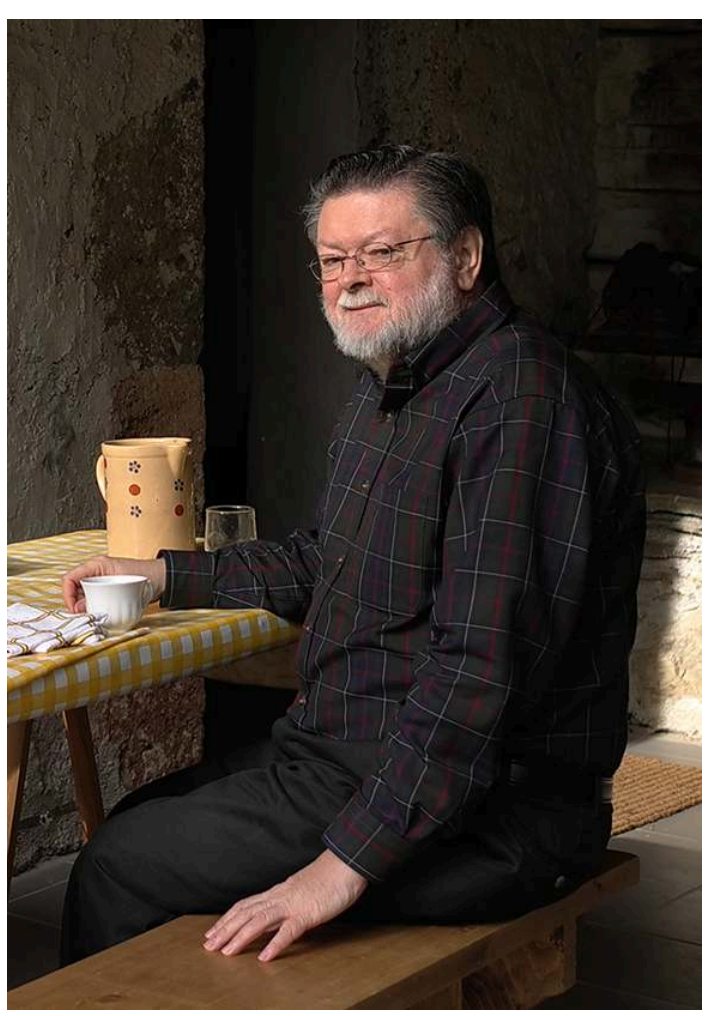

(photo E. Désveaux)

1 Meilleur connaisseur de la constellation ethnique que représentent les Sioux, l'anthropologue Raymond J. DeMallie est décédé le 25 avril 2021, à l'âge de 74 ans. De fait, 
son savoir et sa vigilance intellectuelle embrassaient bien au-delà des Sioux, s'étendant à toutes les Plaines - il fut le maître d'œuvre du Handbook of North American Indians consacré à cette immense aire culturelle ${ }^{1}$ - et jusqu'à un certain point à toute l'ethnographie nord-américaine. Il avait ainsi fondé en 1985 à l'université de l'Indiana à Bloomington, où il avait été recruté dès 1973, l'American Indian Studies Research Institute, qu'il a dirigé en binôme avec Douglas R. Parks, linguiste lui aussi et spécialiste des Plaines, jusqu'à ce qu'une attaque cérébrale, à l'automne 2014, le laisse presque entièrement paralysé et le prive de l'usage de la parole. Bien que suivant et comprenant tout des conversations qui l'entouraient, il en a été réduit à seulement exprimer son accord ou son désaccord. Étrange ironie du sort, car Ray DeMallie était probablement l'universitaire américain qui s'exprimait à l'oral avec le plus de clarté et d'élégance que nous n'ayons jamais, l'un et l'autre, entendu. Son style écrit possédait cette même qualité, quand bien même il abordait les questions les plus complexes de l'organisation sociale ou du système rituel et de leurs évolutions dans le temps. Ray était l'incarnation même de cette figure que nous avons tous en nous du collègue américain idéal : érudit, rigoureux, généreux de ses connaissances, curieux de vos propres idées, accueillant en toutes circonstances, dépourvu de toute arrogance. Bon vivant aussi. Et effectivement, nous (ED et $\mathrm{GH}$ ) avons été reçus à Bloomington année après année avec toutes les marques d'un réel enthousiasme pour nos travaux respectifs. L'American Indian Studies Research Institute a également reçu et soutenu nos étudiants nord-américanistes, à l'instar de Matthieu Charle et de Thierry Veyrié. De façon symétrique, durant plus d'une décennie, Raymond J. DeMallie et Douglas $R$. Parks sont également venus régulièrement à Paris, leur année universitaire bouclée, en juin ${ }^{2}$. Ray intervenait toujours alors dans mon (ED) séminaire, ainsi que parfois dans quelques autres, et c'était certainement un des plus beaux couronnements de mon propre enseignement que je pouvais offrir à mes étudiants. Ensuite, de retour aux États-Unis, Ray and Doug prenaient la route - parfois l'avion aussi - pour les réserves indiennes de Pine Ridge ou de Fort Berthold. Il s'agissait pour eux, les dernières années, non pas tant de faire du terrain que d'encourager et d'accompagner les efforts locaux en matière de revitalisation culturelle et linguistique. Leur aura était immense dans ces communautés.

2 Nul doute que le destin de Ray DeMallie ait été d'exception, dans le sens de celui qui déjoue tout déterminisme bourdieusien. Il est né en 1946 dans une famille modeste de Rochester. Rochester, Upstate New York, appartient à l'époque à Kodak, comme Détroit à General Motors. Mais c'est également la ville de Morgan, l'auteur de Consanguinity and Affinity in the Human Family, l'ouvrage fondateur de l'anthropologie de la parenté, et une ville qui est implantée en plein cœur du territoire iroquois. Ici, les Indiens ont pourtant été relégués en arrière-plan par l'histoire. Leur domaine est devenu une vaste zone industrielle où prédomine la chimie. L'industrie photographique, afin d'offrir à chaque famille américaine l'image de son bonheur encadrée à moindre frais dans le salon, y dévore l'espace sans oublier d'en polluer le ciel, le sol et les rivières. Dès lors, le jeune Ray DeMallie regarde ailleurs : vers les Indiens des grandes plaines, là-bas à l'Ouest où le ciel ne connaît aucune limite. Il s'identifie aux Sioux, là où ses camarades de jeux assumaient volontiers le rôle des vilains cow-boys tueurs d'Indiens. Il se prend de passion. Il va de bibliothèques en libraires spécialisés dans les livres d'occasion en quête d'ouvrages qui leur sont consacrés, tel Black Elk Speaks, de John G. Neihardt, tout en développant au passage un penchant de collectionneur qui ne se démentira jamais chez lui. Ainsi la grande maison qu'il partage avec Doug, située dans les bois à quelques kilomètres du campus de Bloomington, deviendra certainement au fil du temps la plus 
grande bibliothèque privée du monde sur les Indiens des Plaines et l'histoire de l'Ouest américain. Véritable caverne d'Ali Baba, elle recèle en outre une large collection d'objets ethnographiques et des tableaux d'artistes amérindiens renommés (sans omettre une honorable cave à vins).

3 Selon Ray DeMallie lui-même ${ }^{3}$, c'est une conférence prononcée en 1964 à l'université de Rochester par Fred Eggan qui sera doublement décisive pour son orientation future. Eggan, alors le grand Manitou du département d'anthropologie à l'université de Chicago et disciple de Radcliffe-Brown, y exposait qu'au fond la parenté sioux restait un problème largement irrésolu. On se souvient qu'Eggan avait dirigé l'ouvrage collectif The Social Anthropology of the North American Indian Tribes (1937). Le projet se voulait alors en rupture avec le culturalisme boasien et visait à produire une étude des terminologies de parenté des Indiens des Plaines, du Sud-Est et du Sud-Ouest d'obédience fonctionnaliste. Or, si la plupart des grands groupes y étaient traités - tels les Cheyennes, les Fox (renommés depuis Meskwakie), les Apaches, ou encore les Cherokee-, en revanche les Sioux brillaient par leur absence de ce recueil. La voie est toute tracée. Ray se rendra à Chicago et se vouera à élucider l'énigme de la parenté sioux. Il aimait raconter l'anecdote suivante: en 1965, il fut chargé, comme "freshman", d'aller accueillir Claude Lévi-Strauss à l'aéroport, invité alors à prononcer une conférence par le département d'anthropologie de l'université de Chicago. Dès 1966, il se rend dans le Dakota du Sud pour son premier terrain - il travaillera principalement sur la réserve de Cheyenne River. Mais, en parallèle, pressentant le rôle de l'histoire dans la variation des données dont il dispose, il fréquente avec assiduité les archives conservées à Washington, où il se lie avec William C. Sturtevant qui, de son bureau de la Smithsonian Institution, se préparait à lancer la seconde série du Handbook of North American Indians.

Dans un délicieux article publié dans un ouvrage en hommage à l'auteur d'American Kinship (The Cultural Analysis of Kinship. The Legacy of David M. Schneider) ${ }^{4}$, DeMallie raconte avec un humour pince sans-rire un épisode crucial de cette période initiatique. Il relate une séance de son comité de thèse ${ }^{5}$, où, sous la présidence d'Eggan, il se trouve pris en étau entre Raymond Fogelson et David Schneider, soit entre un anthropologue " classique " qui tente tant bien que mal de concilier culturalisme et fonctionnalisme, d'un côté, et, de l'autre, l'iconoclaste bien connu pour lequel la parenté n'est jamais qu'une illusion théorique. Nul doute qu'il aurait été risqué de se déclarer disciple de Schneider dans ces conditions... Et pourtant, comme Ray DeMallie le dit, la leçon de ce dernier est peut-être celle qui l'inspirera le plus, qui énonçait déjà le principe que la parenté reflétait avant tout un « espace sémantique ». À un niveau plus concret, face à un système singulièrement rebelle à l'analyse ${ }^{6}$, tant fonctionnelle que computationnelle, la parenté des Sioux relève bien d'une "solidarité diffuse et persistante » (diffuse, enduring solidarity).

Dans ces années, DeMallie a eu également l'occasion de rencontrer Vine Deloria Jr., jeune auteur issu d'une prestigieuse lignée d'intellectuels sioux ${ }^{7}$ et qu'a rendu célèbre son ouvrage Custer Died For Your Sins (1969), le premier pamphlet résolument postcolonial jeté à la face de l'Amérique bien-pensante. Il prend conscience que la recherche ethnographique ne peut plus désormais plaider l'innocence, du moins du point de vue des autochtones. Elle doit être impliquée. Il ne fait guère de doute que cela l'amena à réviser en partie sa pratique ethnographique, dans le sens d'une plus grande prudence. Ce qui n'allait pas nécessairement à l'encontre de son tempérament, au demeurant. Du coup, 
on doit se demander si la plus grande rencontre que fait Ray DeMallie dans son existence n'est pas celle de James Walker, ou plus exactement des archives laissées par ce dernier.

Walker était un médecin, affecté par le gouvernement fédéral en 1896 à la réserve de Pine Ridge. Il y restera dix-huit ans, jusqu'en 1914, s'improvisant ethnographe, mémorialiste, mais également à la fois greffier et archiviste des Sioux - ces derniers se montrant souvent fascinés par l'écriture, et parfois même enclins à s'y adonner. Développant une vraie complicité avec les vieux guerriers sioux brisés par la Pax americana et la vie misérable de la réserve, il amasse une documentation considérable, comptant plus d'un millier de pages. Ces documents dormaient dans le grenier de la petite-fille de Walker à Denver depuis presque un demi-siècle. Une fois redécouverts, ces Walker's Papers acquirent rapidement un statut presque mythique dans les différents cercles de la siouologie, sans que beaucoup de personnes ne les aient vraiment lus. DeMallie, en compagnie de Elaine Jahner (1942-2003), une nonne bénédictine défroquée, s'attelle à leur publication. Les documents ressortent en gros de trois catégories: des transcriptions d'entretiens, des notes de synthèse ethnographique rédigées par Walker lui-même sur tel ou tel thème (ainsi les modalités du mariage ou les séquences particulières de la danse $d u$ soleil), et enfin des textes rédigés, dans leur langue, par les Lakota eux-mêmes, car certains d'entre eux savaient écrire. Le travail est immense : il faut classer par thème ou par "informateur" les documents; il faut traduire - c'est à cette occasion que Ray approfondira considérablement sa connaissance de la langue sioux -, il faut restituer une intelligibilité à un texte souvent obscur, sans en altérer la teneur. La sensibilité de DeMallie et de Jahner opère une sorte de miracle: la production d'un ensemble qui semble ressusciter sous nos yeux, par l'écrit, la voie des anciens chamans et la poésie profonde de leur discours sur l'implication, par le truchement d'une action rituelle synonyme d'humilité, de l'être humain (en l'occurrence ici le sioux) dans ce monde. On assiste en quelque sorte à l'émergence de la mystique sioux que l'on retrouvera magnifiée quelques années plus tard chez un Black Elk $^{8}$ et qui se substitue à l'éthos résolument guerrier qui caractérisait encore la culture sioux au cœur du XIX ${ }^{\mathrm{e}}$ siècle. Mais les Walker's Papers fourmillent aussi, bien évidemment, d'indications sur cet éthos guerrier. Ils reflètent ainsi une culture en transition, et non pas en phase d'acculturation (celle-ci interviendra plus tard). Si les voix qui émergent des Walker's Papers sont certes personnalisées - dans la plupart des cas, on sait qui parle ou qui écrit -, elles renvoient cependant à une culture commune, quoi qu'en recomposition et ne pouvant donc s'intégrer ni dans le schéma heuristique du fonctionnalisme, ni même dans celui, figé, du culturalisme boasien. Ray DeMallie en prend subrepticement conscience. En ce sens, l'expérience des Walker's Papers conduit à sa conversion à l'ethnohistoire, au primat d'une grille chronologique dans l'interprétation. La mue est toutefois rendue possible par le fait que les textes eux-mêmes semblent nous faire entendre les Sioux des années $1900 \mathrm{au}$ plus près de leur intimité psychique. Par là même, la nature profondément dialogique de la relation ethnographique se maintient. DeMallie est Walker ${ }^{9}$. Il cohabite avec Little Wound, avec George Sword, avec Afraid of Bear ou encore avec Antoine Herman, les frères Nines ou Thomas Tyon, les interprètes privilégiés du médecin devenu, par compassion, terme entendu dans son sens le plus fort, l'ethnographe de référence des Sioux.

7 On comprend ainsi comment Ray DeMallie se fait de plus en plus l'avocat de l'ethnohistoire ${ }^{10}$ - qu'il enseigne à Bloomington à partir de $1976-$, conçue comme une méthode susceptible de mieux faire valoir les perspectives amérindiennes, en croisant terrain ethnographique, sources coloniales et récits autochtones, dans leurs langues 
respectives. Il s'agit d'écrire une histoire des Indiens qui, en rupture avec l'historiographie américaine, incorpore leurs conceptions culturelles; au fond, d'écrire des ethnographies historiques. Ray DeMallie ne s'engagera jamais dans l'écriture d'une histoire des Sioux, car il en a estimé les bases documentaires, sur le plan anthropologique, toujours trop fragiles. Mais, à travers ses multiples travaux, il offre des interprétations novatrices sur la vision amérindienne des traités conclus avec les autorités américaines, sur la Ghost Dance, ou encore sur la bataille de Little Big Horn ${ }^{11}$. Lors d'un colloque à Paris en juin 2014, il ferraille avec l'historien Richard White, leur discussion faisant ressortir deux visions totalement contrastées dans la façon d'écrire l'histoire des Indiens. Face à une interprétation de l'Amérindien en proto homo economicus, DeMallie insiste de son côté sur la nécessité d'une histoire foncièrement inscrite dans la culture des acteurs ${ }^{12}$. Claude Lévi-Strauss, à l'inverse, pointera dans le compte rendu de son Handbook sur les Plaines, ce qu'il considère comme une dérive historiciste de l'anthropologie américaine par rapport à ses illustres fondateurs ${ }^{13}$.

Par son inlassable travail d'édition de textes restés jusqu'alors dans les archives, DeMallie reconnaît toute l'importance des relations françaises. Elles constituent en effet, à partir du XVII ${ }^{e}$ siècle, la première strate de documentation sur les Sioux ${ }^{14}$. Cette immersion dans les textes français conforte la discrète francophilie de Ray, dont le patronyme est d'origine française - son ascendance hollandaise laisse penser qu'il avait des ancêtres huguenots -, et qui avait appris la langue de Molière au lycée. Son intérêt pour cette dernière, ainsi que pour l'anthropologie comme l'histoire à la française, contribue du reste à sa profonde singularité dans le paysage intellectuel américain. Il n'empêche qu'il était aussi l'un des derniers représentants d'une longue lignée d'anthropologues américains -hommes et femmes-, héritiers des premiers collaborateurs de la Smithsonian Institution ${ }^{15}$, pour lesquels les autochtones de leur pays méritaient toute l'attention possible, tant leurs cultures et leurs langues se présentaient d'emblée avec tous les attributs de la sophistication, mais se révélaient également d'une tragique fragilité. 
Fig. 2 - Douglas R. Parks, Paris, 2014

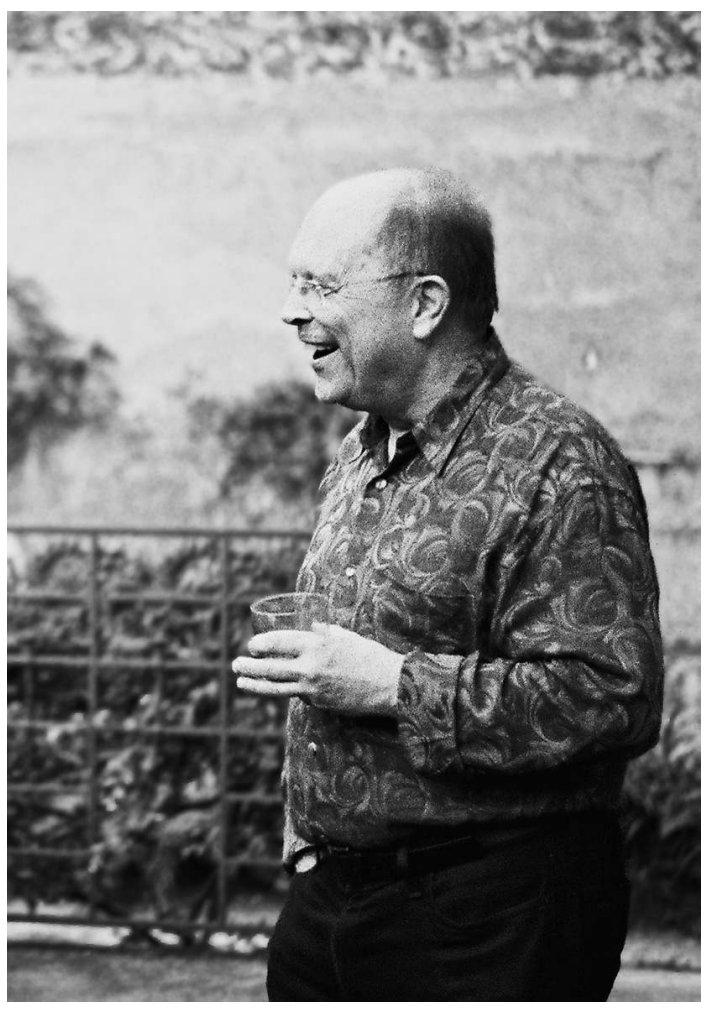

(archives personnelles d'E. Désveaux)

9 Au moment où s'achève l'écriture de cet hommage, nous apprenons le décès de Douglas R. Parks (20 mai 2021), compagnon d'armes de Ray DeMallie dans l'étude des Indiens des Plaines. Né à Long Beach (Californie) en 1942, Doug Parks a été formé à la linguistique à l'université de Californie à Berkeley où, sous l'influence de Mary R. Haas, une élève de Sapir, il trouve sa vocation : documenter les langues indiennes des Plaines, qu'il s'agisse de valoriser des récits recueillis par ses prédécesseurs - tels ceux du chamane pawnee Roaming Scout, transcrits par James R. Murie et George A. Dorsey - ou d'interviewer de vieux conteurs avant leur disparition. Quand il débute son terrain en Oklahoma chez les Pawnee, en 1965, il ne reste que 250 locuteurs ; cinq ans plus tard, alors qu'il entame son travail de collecte dans la réserve de Fort Berthold (Dakota du Nord), Parks identifie 200 locuteurs arikara, dont une vingtaine se souviennent de récits traditionnels. Il noue alors des liens étroits avec les Indiens, s'appuyant sur quelques informateurs ou informatrices privilégiés, à commencer par Lula Nora Pratt (1908-2001) chez les Pawnee, ou Alfred Morsette Sr. (1911-1989) chez les Arikara.

Grand spécialiste du pawnee et de l'arikara, deux langues de la branche septentrionale de la famille caddoane, Parks, pour qui la connaissance des langues est un préalable à l'écriture d'une histoire des Indiens, apprend puis enseigne également le lakota, l'assiniboine et l'hidatsa, trois langues sioux. C'est lui qui rédige toutes les sections ethnonymiques du Handbook of North American Indians consacré aux Plaines, et qui en assure plus généralement la coordination linguistique.

$11 \mathrm{Au}$ cours de sa carrière, il obtient successivement un poste à l'université de l'Idaho (1968-1973), puis au Mary College de Bismarck (1974-1982) - où il dirige le programme linguistique qu'avait initié Elaine Jahner-, et enfin à l'université de l'Indiana à Bloomington (1983-2019), en tant que professeur, où il rejoint donc Ray DeMallie. 
Travailleur infatigable, il édite des centaines de récits qu'il a recueillis et traduits ${ }^{16}$, rédige une grammaire ${ }^{17}$ et des dictionnaires ${ }^{18}$. Il élabore en parallèle des matériaux didactiques en vue de faciliter l'enseignement de langues en perdition dans l'optique de leur revitalisation ${ }^{19}$. Le tournant des années 2000 voit en effet la disparition des derniers locuteurs du pawnee et de l'arikara. Grâce au travail de Parks, ces deux langues font toutefois aujourd'hui l'objet d'un enseignement systématique dans les écoles des réserves. Doug était un personnage haut en couleurs, trouvant toujours à raconter avec humour, selon ses interlocuteurs ou les circonstances, telle ou telle anecdote. Nul doute que ce tempérament jovial a servi ses desseins auprès des Indiens dans les réserves, comme auprès de ses étudiants à l'université. Ses efforts indéfectibles en vue de préserver le patrimoine linguistique et narratologique des Grandes Plaines nordaméricaines méritent tout notre respect. Parmi les Arikara, qui l'avaient adopté, il était connu sous le nom de White Crow (kaakaataaká).

\section{NOTES}

1. Raymond J. DEMALLIE (dir.), Handbook of North American Indians, 13. Plains, Smithsonian Institution, Washington, 2001, 2 vol.

2. En 2002-2003, DeMallie est invité à l'École des hautes études en sciences sociales où il occupe la French-American Foundation Chair in American Civilization. Il passe toute l'année académique à Paris et c'est à partir de ce moment qu'il prend l'habitude d'y retourner régulièrement.

3. Voir son article : Raymond J. DEMALLIE, «Procrustes and the Sioux: David M. Schneider and the study of Sioux Kinship ", in Richard Feinberg et Martin Ottenheimer (dir.), The Cultural Analysis of Kinship. The Legacy of David M. Schneider, University of Illinois Press, Urbana, 2001, p. 46-59.

4. Raymond J. DEMALLIE, « Procrustes and the Sioux... », op. cit.

5. Son Ph.D., décroché en décembre 1971, est intitulé Teton Dakota Kinship and Social Organization.

6. Voir Emmanuel DéSVEAUX, «Parenté, rituel, organisation sociale : le cas des Sioux », Journal de la Société des américanistes, 83, 1997, p.111-140; repris dans La Parole et la substance. Anthropologie comparée de l'Amérique et de l'Europe, Les Indes Savantes, Paris, 2017, p. 61-93.

7. Leur première rencontre intervient au congrès de l'American Anthropological Association à San Diego en 1970: ce fut le début d'une longue collaboration entre les deux hommes. Vine Deloria Jr. (1933-2005) est le neveu de Ella C. Deloria, que DeMallie rencontra en 1965, et le fils d'un prêtre épiscopalien, Vine Deloria Sr., qui, à partir de 1969, initia DeMallie à la langue lakota. Le nom Deloria dérive de Deslauriers, nom d'un coureur de bois francophone qui fit souche chez les Lakota dans les années 1820 . Voir Raymond J. DEMALLIE, « De Deslauriers à Deloria. L'identité française d'une famille 
sioux ", in Gilles Havard et Mickaël Augeron (dir.), Un Continent en partage. Cinq siècles de rencontres entre Amérindiens et Français, Les Indes Savantes, Paris, 2013, p. 535-558.

8. Black Elk Speaks. Being the Life Story of a Holy Man of the Oglala Sioux, raconté par John G. Neihardt [Flaming Rainbow], annoté par Raymond J. DeMallie, avec des illustrations de Standing Bear, Excelsior Editions/State University of New York Press, Albany, 2008.

9. Les Walker's Papers ont donné lieu à trois publications distinctes : Raymond J. DEMALLIE et Elaine A. JAHNER (dir.), Lakota Belief and Ritual, University of Nebraska Press, Lincoln, 1980 ; Raymond J. DEMALLIE (dir.), Lakota Society, University of Nebraska Press, Lincoln, 1982 ; Elaine A. JAHNER (dir.), Lakota Myths, University of Nebraska Press, Lincoln, 1983. Voir notre compte rendu du premier volume : Emmanuel DÉsVEAUX, « J. R. Walker, Lakota Belief and Ritual » [compte-rendu], L'Homme, 22 (3), 1982, p. 124-125; et celui de Claude Lévi-Strauss lors de la réédition de l'ouvrage au format de poche: «P. Holder, The Hoe and the Horse on the Plains. A Study of Cultural Development among North American Indians J. G. Neihardt, When the Tree Flowered. The Story of Eagle Voice, a Sioux Indian - J. R. Walker, Lakota Belief and Ritual » [compte-rendu], L'Homme, 32 (122-124), 1992, p. 452-453.

10. Voir Sebastian Felix BRAUN (dir.), Transforming Ethnohistories. Narrative, Meaning, and Community (University of Oklahoma Press, Norman, 2013), en particulier le chapitre de David Reed MILLER, « Borders and Layers, Symbols and Meanings: Raymond J. DeMallie's Commitment to Ethnohistory, with Nods to Thick Description and Symbolic Anthropology », p. 23-42.

11. Voir notamment "These Have No Ears: narrative and the ethnohistorical method", Ethnohistory, 40 (4), 1993, p. 515-538, son article « définitif » sur l'ethnohistoire.

12. Cette communication, faite lors du colloque du $30^{\mathrm{e}}$ anniversaire du Centre d'études nord-américaines (EHESS), fera l'objet de sa dernière publication : Raymond J. DEMALLIE et Gilles HAVARD, «Writing the History of North America from Indian Country: the view from the North-central Plains, 1800-1870 », Journal de la Société des américanistes, 105 (1), 2019, p. 13-40.

13. Claude LÉVI-STRAUSS, « Raymond J. DeMallie, Handbook of North American Indians. 13 : Plains » [compte-rendu], L'Homme, 164, 2002, p. 167-169.

14. La publication des écrits du coureur de bois Jean-Baptiste Truteau, en collaboration avec Doug Parks et le linguiste québécois Robert Vézina, couronne cette entreprise de collecte et d'édition : Raymond J. DEMALLIE, Douglas R. PARKS et Robert VÉZINA (dir.), A fur trader on the Upper Missouri. The Journal and Description of Jean-Baptiste Truteau, 1794-1796, University of Nebraska Press, Lincoln, 2017.

15. Voir Claude LÉVI-STRAUSS, «L'œuvre du Bureau of American Ethnology et ses leçons », in Anthropologie structurale Deux, Plon, Paris, 1973, p. 63-75.

16. Douglas R. PARKS, Traditional Narratives of the Arikara Indians, University of Nebraska Press, Lincoln, 1991, 4 vol.

17. Douglas R. PARKS, A Grammar of Pawnee, Garland Publishing, Inc., New York, 1976.

18. Douglas R. PARKS, An English-Arikara Student Dictionary, White Shield School District, Roseglen, 1986; Douglas R. PARKS et Lula Nora PRATT, A Dictionary of Skiri Pawnee, University of Nebraska Press, Lincoln, 2008.

19. Douglas R. PARKS, Janet BELTRAN et Ella P. WATERS, An Introduction to the Arikara Language, White Shield School District, Roseglen, 1998-2002, 3 vol. ; Douglas R. PARKS, An Introduction to the Pawnee Language. A Set of Multimedia Lessons, American Indian Studies 
Research Institute, Bloomington, 2001, 2 vol. ; Douglas R. PARKS, Salena DITMAR et Mindy J. MORGAN, Nakoda Language Lessons. Preliminary Edition, Belknap College, Fort Belknap/ American Indian Studies Research Institute, Indiana University, Bloomington, 1999.

\section{AUTEURS}

EMMANUEL DÉSVEAUX

EHESS

GILLES HAVARD

CNRS 